\title{
SUR UN HASTOSPICULUM PARASITE DU CROTALE
}

\author{
Par C. DESPORTES
}

En r $9_{2} 3$, J. Skrjabin créait le genre Hastospiculum pour une filaire du varan, H. varani, trouvée au Turkestan. Dans les années qui suivirent, plusieurs filaires, également parasites de varans, furent placées dans ce nouveau genre : elles étaient au nombre de cinq espèces, dont deux décrites bien antérieurement au travail de Skrjabin sous le nom générique de Filaria $s$. l., lorsqu'en I933, Chitwood faisait connaître deux nouvelles espèces, $H$. setiferum et $H$. onchocercum, trouvées à la dissection de grands serpents. Ce dernier auteur fait alors la révision de toutes les espèces décrites et, tout en tenant pour fragile la valeur de certaines d'entre elles, propose pour celles-ci une classification.

Cependant, en 1935 , E. E. Wehr met les genres Hastospiculum et Parhamatospiculum en synonymie du genre Hamatospiculum Skrjab. I 9 г 6 : ce dernier genre comprend alors 8 espèces parasites d'oiseaux et 7 de reptiles. J. Skrjabin, qui avait défini chacun des trois genres Hamatospiculum, Parhamatospiculum et Hastospiculum, ne semble pas partager cette conception et, quelques mois plus tard, nous voyons figurer, dans la classification des Filariata qu'il publie avec N. P. Schikhobalova, ces trois mèmes genres présentant chacun des caractères parfaitement déterminés. En réalité, la valeur de ces caractères ne paraît pas entièrement fondée : ce qui tient pour la plus grande part au manque de renseignements sur les différentes espèces, en particulier sur celles appartenant au genre Hastospiculum.

C'est pourquoi nous avons cru qu'il pouvait être intéressant, pour la connaissance de ces vers, de publier l'étude que nous avons faite de filaires du Crotale, récoltées par notre excellent ami, le $\mathrm{D}^{\mathrm{r}} \mathrm{J}$. Callot, au cours de la fructueuse mission qu'il a effectuée au Brésil.

Ces filaires, trois mâles, dont un en parfait état, et quelques femelles, dont une complète, étaient logées dans le tissu cellulaire sous-cutané et dans les séreuses entourant la partie postérieure de l'intestin d'un Serpent à sonnette, Crotalus terrificus Laur., de I m. 25 , capturé à São Laurenço, à 15 kilomètres à l'Ouest de Recife. ANNALES DE PARAsitrologie, T. XVIII, Nos 4-5-6. - r94r, p. 198-208. 
J. Callot nous apprend que le nombre de ces nématodes pouvait être d'une vingtaine, certains atteignant de 60 à $80 \mathrm{~cm}$. et renfermant des œuts embryonnés, d'autres étant de plus petite taille (fig. I).

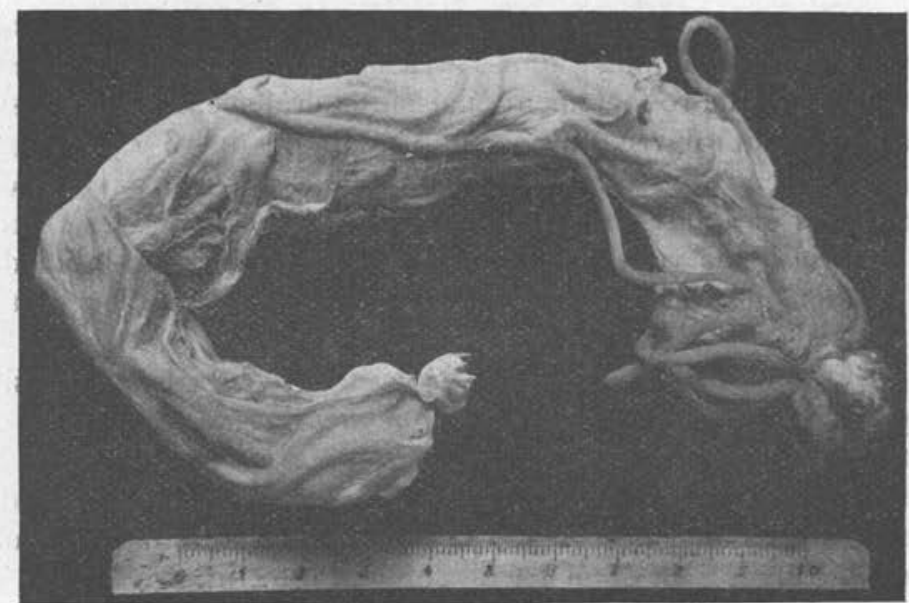

Fıc. I. - Péritoine entourant une portion d'intestin de Crotalus terrificus et envahi par Hastospiculum onchocercum Chit. 1933 forma major.

\section{Description}

Mâle. - Les trois exemplaires mâles récoltés mesuraient respectivement 72,68 et $58 \mathrm{~mm}$.; le diamètre maximum du corps des deux premiers était de o $\mathrm{mm}$. 65 , celui du plus petit de $0 \mathrm{~mm}$. 58 .

Le corps blanchâtre, régulièrement cylindrique, est terminé par une queue plus grêle, enroulée vers la partie ventrale.

La cuticule présente une annulation particulière très nette sur tout le corps, se continuant sur la partie dorsale de la queue, mais disparaissant sur la partie ventrale de celle-ci et, antérieurement, au niveau de l'anneau nerveux. Cette annulation est constituée par des épaississements linéaires de la cuticule, larges de $2 \mu .5$ au milieu du corps, où ils sont séparés les uns des autres par des intervalles de 20 à $25 \mu$, plus grêles et plus rapprochés vers les deux extrémités du ver (intervalles de $3 \mu$ à la naissance des ailes caudales). Ces stries n'entourent pas complètement le corps, mais sont interrompues, déviées ou dichotomisées au milieu de chaque champ latéral, et ceci de telle manière 
què les stries constituant l'annulation de la moitié dorsale du corps se mettent en rapport avec celles de l'annulation ventrale. On peut également observer, en dehors des champs latéraux, l'anastomose ou l'interruption des stries, mais ceci est plus rare.

Les champs latéraux, qui ont une largeur de $50 \mu$ au milieu du corps, occupent chacun environ un quarantième de sa circonférence.

L'orifice buccal, allongé dans le sens dorso-ventral, est entouré d'une zone cuticulaire fortement épaissie latéralement pour constituer de chaque côtê une lèvre plus ou moins cubique de $\mathrm{r} 3 \mu$ de hauteur; les formations en épaulettes, seulement visibles en vue frontale, portent les amphides et quatre des huit papilles submédianes: les deux plus dorsales et les plus ventrales qui sont très basses; les submédianes latérales sont au contraire nettement bombées; toutes les huit sont larges.

Les papilles cervicales (dierids), légèrement ventrales, sont situées à $442 \mu$ de l'extrémité antérieure ; l'anneau nerveux se trouve à $215 \mu$ de cette extrémité.

L'œsophage, de $8 \mathrm{~mm}$. environ, est en deux parties : sa portion antérieure musculaire mesure $350 \mu$; sa portion glandulaire, régulièrement cylindrique, a un diamètre de $300 \mu$; l'intestin, qui lui fait suite, ést à peu près rectiligne (diamètre : $170 \mu$ ).

L'extrémité caudale conique porte des ailes bien développées qui entourent largement la pointe (longueur de l'aile : $400 \mu$, largeur roo $\mu$ environ). Chaque aile porte à sa face interne et dans sa partie postérieure un pli vertical convexe : les deux plis droit et gauche délimitent alors une sorte de poche qui entoure l'extrême pointe du ver. Supportant les ailes, existent quatre paires de papilles préanales pédonculées, de $80 \mu$ de hauteur; plus en arrière se trouve une paire de papilles semblables, adanales; à I $40 \mu$ de l'extrémité caudale, de part et d'autre de l'orifice cloacal, existent en outre deux organes papilliformes couchés latéralement et limitant de chaque côté un bourrelet réniforme épais qui borde postérieurement l'orifice cloacal; en arrière des plis alaires précédemment décrits, on observe encore une paire de petites papilles pédonculées postanales, puis au voisinage de chacune d'elles débouche au sommet d'une minuscule saillie un fin conduit ; postérieurement, apparaît un second bourrelet médian transversal, à sommet caréné, et dont les deux extrémités se terminent par une pointe conique dirigée en arrière ; enfin, plus en arrière encore, se trouvent deux petites papilles sessiles ventro-latérales, bien visibles, situées de part et d'autre de la pointe de la queue, laquelle se présente sous la forme d'une petite saillie circulaire dont la cuticule rugueuse est côtelée dans le sens dorso-ventral. 
L'appareil génital, très simple, est constitué par un testicule antérieur, plaqué contre l'œsophage glandulaire et qui naît à $3 \mathrm{~mm}$. 7 de l'extrémité céphalique : il se prolonge graduellement par un canal déférent à peu près rectiligne sur toute la longueur dú corps, mais

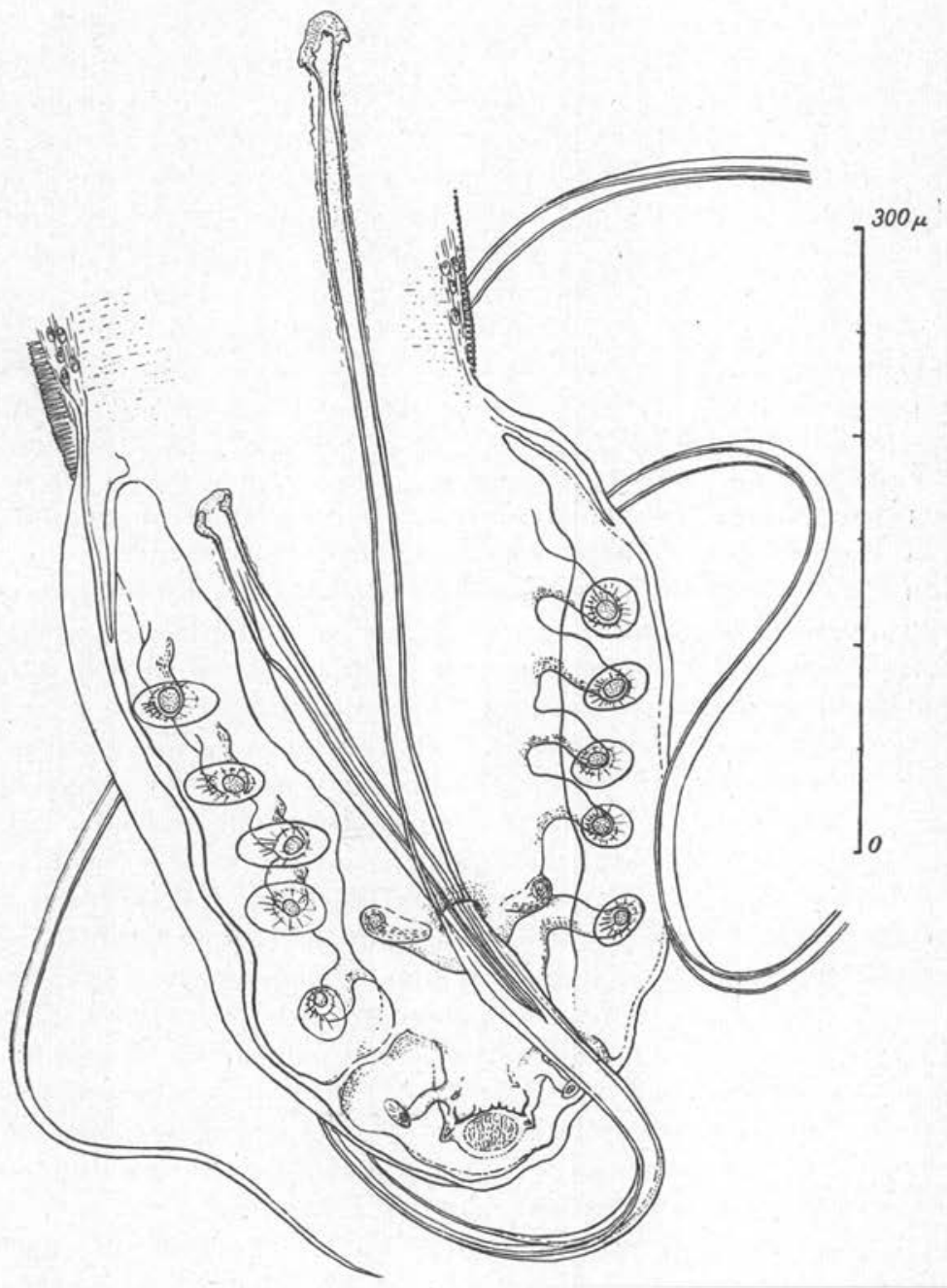

FIG. 2. - Hastospiculum onchocercum Chit. I933 forma major; extrémité caudale du mâle. 
plus ou moins replié vers l'extrémité postérieure. Les spicules, de mème structure mais très inégaux, ont une extrémité proximale renflée suivie d'un col plus ou moins grêle; ils sont tous deux légèrement aplatis dans la partie antérieure dont le diamètre maximum est de I $2 \mu$ pour le spicule gauche, de I $8 \mu$ pour le droit. Le spicule gauche mesure $2 \mathrm{~mm}$. 75 : il porte sur toute sa longueur deux expansions latérales étroites extrèmement minces, mais élargies à $500 \mu$ de l'extrémité proximale en deux fines ailes à bord plus ou moins régulier, et qui semblent constituer une gouttière pour l'extrémité distale du spicule droit (ce que nous avons observé sur les trois mâles examinés). Vers la partie distale, le spicule gauche diminue progressivement pour se terminer en une pointe excessivement fine. Le spicule droit, long de $300 \mu$ environ, présente également deux expansions aliformes, plus larges cependant que celles du spicule gauche; sa portion distale se termine brutalement par une pointe acuminée, un peu tordue. La gaine des spicules est fortement plissée lorsque ceux-ci sont évaginés. Pas de gorgeret (fig. 2).

Femelle. - Le corps cylindrique, rosé, de $634 \mathrm{~mm}$. chez la seule femelle complète, a un diamètre de $2 \mathrm{~mm}$. lorsqu'aucune pression n'est exercée à sa surface : il s'atténue progressivement vers les extrémités, surtout postérieurement. La cuticule présente une annulation identique à celle du mâle : chez la femelle, l'intervalle séparant deux stries consécutives atteint au milieu du corps $6 o \mu$; cet intervalle diminue antérieurement $(25 \mu$ à $8 \mathrm{~mm}$. de la tête et $\mathrm{I} 5 \mu$. à $\mathrm{I} \mathrm{mm}$. de celle-ci), et postérieurement, tout à fait au voisinage de la pointe. L'annulation disparait antérieurement, au voisinage de l'anneau nerveux, et présente une solution de continuité sur les champs latéraux. Ces derniers ont une largeur de $300 \mu$ ( $\mathrm{r} / 20$ de circonférence).

L'orifice buccal ovalaire à grand axe dorso-ventral est limité latéralement par de fortes lèvres denticulaires de $3 o \mu$ de hauteur, $20 \mu$ de largeur, légèrement bilobées au sommet. A la base de chaque lèvre on observe trois indentations sous-cuticulaires.

Les épaulettes sont visibles en vue frontale; les amphides sont larges, centrées par un orifice circulaire; les huit papilles submédianes reposent sur des bases élargies; en avant des submédianes latérales existent des épaississements de la cuticule en forme de lunules, qui bordent les lobes des épaulettes (fig. 3). Les papilles cervicales, situées sur la moitié ventrale des champs latéraux, sont à $440 \mu$ de l'extrémité antérieure; l'anneau nerveux se trouve sensiblement à la mème distance de cette extrémité.

L'œsophage mesure $\mathrm{ig} \mathrm{mm}$., dont o $\mathrm{mm} .8$ pour la portion muscu- 
laire; le diamètre de sa portion glandulaire cylindrique est de $550 \mu$; il se continue par un intestin légèrement plus grêle, à peu près rectiligne, et fortement comprimé par les tubes utérins. Le tube digestif s'atténue vers son extrémité postérieure qui est atrophiée. L'anus, situé à $75 \mu$ de la pointe, n'est pas fonctionnel : il a l'aspect d'une fente réniforme de $14 \mu$ de longueur située au milieu d'une petite plage dépourvue de stries.

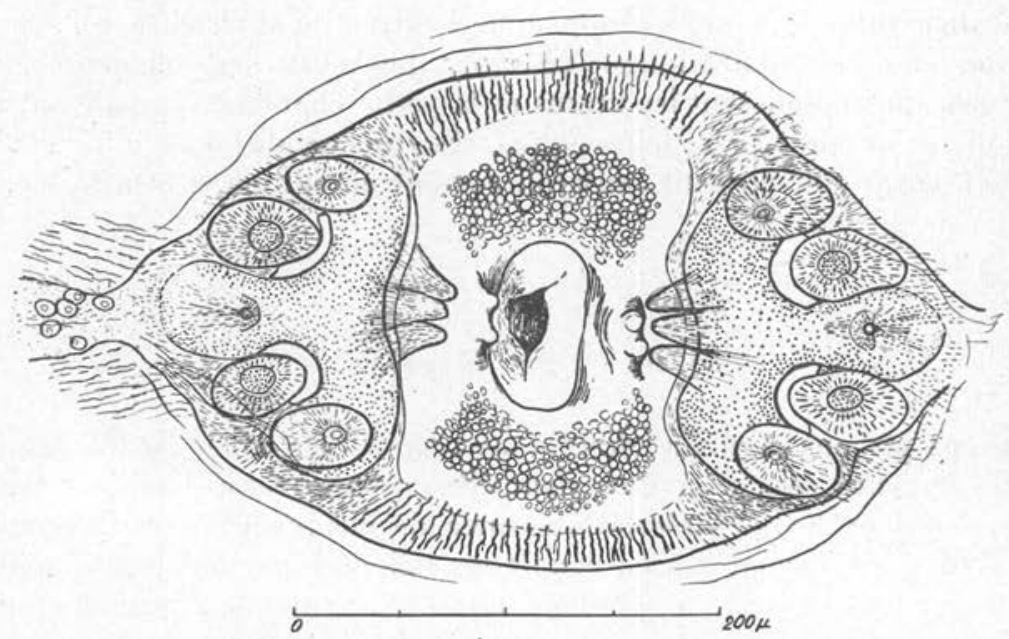

Fic. 3. - Hastospiculum onchocercum Chit. I933 forma major; extrémité céphalique de la femelle en vue frontale.

L'extrémité caudale, arrondie, porte une ornementation particulière très fine : les stries concentriques qui résultent de l'annulation sont en effet interrompues à l'extrême pointe par d'autres stries qui leur sont plus ou moins perpendiculaires et qui entourent deux orifices glandulaires papilliformes situés de part et d'autre d'une saillie médiane ovalaire, un peu dorsale, qui est ornée de fines granulations.

L'appareil génital femelle est du type amphidelphe. L'ovaire antérieur naît à $4 \mathrm{~cm}$. de l'extrémité céphalique, décrit un grand nombre de boucles et se prolonge insensiblement par un oviducte très replié qui atteint la partie antérieure de l'œsophage musculaire, puis, après s'être replié postérieurement, se fusionne à l'utérus antérieur, à I mm. de l'extrémité céphalique. L'ovaire postérieur naît à $\mathrm{I} 2 \mathrm{~cm} .85$ de l'extrémité caudale, remonte légèrement antérieurement de quelques millimètres en décrivant plusieurs boucles, puis se dirige postérieure- 
ment en se continuant par l'oviducte ; ce dernier se fusionne, à $7 \mathrm{~cm} .4$ de l'extrémité caudale, à l'utérus postérieur. Les deux tubes utérins, qui remplissent presque tout le corps du ver, se fusionnent eux-mêmes en un tube unique à $18 \mathrm{~mm}$. de l'extrémité antérieure; les parois de ce conduit s'épaississent alors fortement pour constituer un vagin cylindro-conique de $600 \mu$ de diamètre en son milieu, dirigé antérieurement, sinueux et présentant à sa partie terminale une petite dilatation ampullaire, laquelle renfermait, chez la femelle examinée, un œuf. L'orifice vulvaire, situé à o $\mathrm{mm}$. 9 de l'extrémité antérieure, a l'aspect d'une fente cuticulaire longue de $240 \mu$. Les œufs, sensiblement sphériques, qui remplissent les utérus et la petite chambre vaginale ont un diamètre de 40 à $45 \mu$; leur coque, très translucide (de $2 \mu 5$ d'épaisseur), laisse voir un petit embryon enroulé en spirale et bien formé.

\section{Discussion}

\section{A. - Sur le genre.}

Nous plaçons la filaire dont nous venons de donner la description dans le genre Hastospiculum dont elle présente, outre l'habitus général, les deux lèvres latérales cubiques de chaque còté de la bouche ovalaire, les formations en épaulettes trilobées portant huit papilles submédianes et deux amphides latérales, la cuticule ornée, les ailes caudales du mâle bien développées, fusionnées postérieurement et soutendues par de hautes papilles pédonculées, les spicules très inégaux, l'amphidelphie, la position de la vulve et les œufs embryonnés. Ainsi, nous considérons comme Yorke et Maplestone (1926) cet ensemble de caractères comme suffisant pour définir ce genre et l'opposer notamment au genre Hamatospiculum Skrjab. igı6 (= Parhamatospiculum Skrjab. et Petr. I928) (1) avec lequel il avait été mis en synonymie (E. E. Wehr, I935).

Cependant, pour opposer ces genres, nous croyons que les caractères proposés par Skrjabin et Schikhobalova ( 1936$)$ ne sont pas suffisamment précis. Ces auteurs écrivent en effet :

L'extrémité médiane des formations en épaulettes de chaque côté est munie dans sa partie moyenne de quatre éminences en forme de papilles. Les

(I) Les genres Hamatospiculum Skrjabin 1916 et Parhamatospiculum Skr. et Petr. $x_{928}$, ont été mis en synonymie par Sandground (1933). Cette synonymie est acceptée par Tubangui ( $\left.x_{9} 35\right)$, qui décrit trois nouvelles espèces pour le genre, mais elle n'est pas admise par Skrjabin et Schikhobalova r 936. 
spicules sont dépourvus d'ailes. Parasites des reptiles $=$ Hastospiculum Skrjab. ${ }_{1923}$.

L'extrémité antérieure des formations en épaulettes est pourvue, dans sa partie moyenne, d'une grande papille saillante. Dans la partie moyenne, le spicule est muni de fines ailes impaires. Parasites du tissu sous-cutané des oiseaux $=$ Parhamatospiculum Skr. et Petr. ${ }_{1928}$.

Or, en analysant chaque caractère, nous faisons les remarques suivantes :

$\mathrm{I}^{\circ}$ Les éminences papilliformes (nous pensons comme H. A. Baylis qu'il s'agit là d'insertions musculaires) qui sont au nombre de quatre seulement pour l'espèce $H$. varani, sont au nombre de trois, d'après H. A. Baylis (r93o), chez H. macrophallos, et également de trois, d'après Chandler (r929) et Chitwood ( 1933$)$, chez H. spiniger; nous trouvons ce mème nombre sur nos exemplaires; enfin une seule de ces formations existerait chez $H$. setiferum (Chitwood, I933); elles ne sont pas signalées pour les autres espèces.

$2^{\circ}$ Les papilles saillantes situées à l'extrémité antérieure des formations en épaulettes chez Parhamatospiculum ne sont autres que les lèvres cubiques saillantes d'Haslospiculum : elles sont donc présentes dans les deux genres.

$3^{\circ}$ La présence d'ailes spiculaires, observées chez Hamatospiculum (=P.) nodulosum et chez $H$. $(=P$.) bubicola, signalées encore chez $H$. brasilianum par Yorke et Maplestone $\left(\mathrm{r}_{92} 6\right)$ et chez H. quadridens, par Boulenger ( 1928$)$, n'est pas mentionnée pour les autres espèces. Cependant, nous trouvons des ailes spiculaires étroites chez les trois mâles d'Hastospiculum du crotale. Il ne peut donc s'agir ici d'un caractère différentiel.

C'est pourquoi nous croyons qu'il serait préférable, sans faire appel à la taille des parasites, toujours plus faible chez les Hamatospiculum, ni aux hôtes (actuellement des oiseaux pour Hamatospiculum, des reptiles pour Hastospiculum), de prendre pour caractère différentiel les dimensions des ailes caudales du mâle.

La faible largeur de ces ailes chez les différentes espèces appartenant au genre Hamatospiculum est précisée par G.-L. Boulenger (i 928 ), Sandground ( r $^{33}$ ), Skrjabin et Petrow (r928, г 935 ), Tubangui ( 1935$)$ : elle apparaît également sur les figures de l'extrémité caudale des mâles, données par ces auteurs.

Chez Hastospiculum, ces ailes sont au contraire bien développées et largement fusionnées postérieurement, et, à l'exception du mâle endommagé de $H$. spinigerum, pour lequel Chandler a parlé de " short alae " - quoique ce caractère ne semble pas évident sur la figure- 
qu'il nous en donne - le qualificatif de "large " appliqué à ces ailes caudales par Yorke et Maplestone pour définir le genre, paraît tout à fait justifié.

On remarquera en outre que généralement les grandes papilles caudales soutendent les ailes chez tous les Hastospiculum (à l'exception de $H$. bipinnatum figuré par 0 . Linstow) et qu'elles en sont séparées chez les Hamatospiculum (excepté chez H. pertenuialatum, d'après Sandground).

Ajoutons enfin que nous ne considérons pas, du moins à l'heure actuelle, comme étant un caractère différentiel important, les deux saillies médianes situées en arrière du cloaque d'Hastospiculum, seulement signalées chez $H$. gouldi par Yorke et Maplestone (1926) et observées sur nos exemplaires. Nous spécifions alors que celle de ces saillies qui est postérieure se termine par deux pointes coniques en forme de papilles, ce qui expliquerait la figure donnée par Chitwood de l'extrémité caudale de $H$. onchocercum. Existent-elles chez les autres espèces ? Nous ne pouvons le préciser, mais n'oublions pas que chez deux espèces du genre Hamatospiculum (H. nodulosum et $H$. bubicola) existe également une formation médiane en arrière du cloaque (Skrjabin et Petrow, r $9^{2} 8$ et r 935 ).

\section{B. - Sur l'espèce.}

D'après la forme et le diamètre du spicule gauche, on peut répartir, comme Chitwood, les différentes espèces du genre Hastospiculum en deux groupes, le premier renfermant celles dont le spicule gauche est aussi large que le droit et d'un diamètre à peu près constant sur toute sa longueur : telles seraient $H$. bipinnatum (Linst. 1899), H. varanı Skrj. ${ }^{1} 9_{2} 3$ (synonyme de $H$. macrophallos pour Sandground), H. spinigerum Chandler r 929 (considéré par Baylis comme identique à $H$. macrophallos) et $H$. macrophallos (Parona $\mathrm{I} 889$ ). S'opposant à ce premier groupe, les espèces constituant le second ont un spicule gauche très effilé : ce caractère, observé chez $H$. gouldi Yorke et Maplestone $19^{26}$, se retrouve sur la figure donnée par Chitwood de H. setiferum Chit. I935; il est précisé par cet auteur dans la description de $H$. onchocercum Chit. i 933 .

La filaire étudiée dans le présent travail se place également dans le second groupe, et, poussant plus loin l'identification de ce nématode, nous pouvons nous rendre compte qu'aucun des caractères - si ce n'est la longueur du corps des vers $\left(\sigma^{x}=58.72 \mathrm{~mm}\right.$.; $q=$ $634 \mathrm{~mm}$.) - ne peut être invoqué d'une manière absolue pour consi- 
dérer la filaire du crotale comme une espèce nouvelle et la séparer de $H$. onchoćercum $\left(\sigma^{x}=38-55 \mathrm{~mm}\right.$. ; $ᄋ=170-350 \mathrm{~mm}$.) : mêrme disposition des formations en épaulettes, des lèvres cubiques épaisses, des papilles submédianes, des amphides (également saillantes dans les deux cas), mèmes particularités de l'annulation, de ses anastomoses et des solutions de continuité des stries (nous précisons que l'interruption de celles-ci siège sur les champs latéraux); même position antérieure de la vulve; dimension sensiblement égale des œufs. Enfin, si la description que nous donnons de l'extrémité caudale de nos exemplaires mâles ne semble pas coïncider avec celle, peut-être un peu condensée, que donne Chitwood de $H$. onchocercum, il est cependant remarquable de voir à quel point la figure précise qu'en donne cet auteur et la nôtre sont semblables. Aussi croyons-nous qu'il n'y a pas lieu de considérer la filaire du serpent à sonnette du Brésil comme appartenant à une espèce nouvelle, différente de celle d'un boa des mêmes régions; tout au plus devons-nous la nommer Hastospiculam onchocercum Chit. 1933 forma major, puisque dans la clef pour définir les espèces du genre Hastospiculum, Chitwood s'appuyait précisément sur les dimensions des vers pour séparer $H$. setiferum et $H$. onchocercum.

\section{RÉSUMÉ}

Nous décrivons une grande filaire récoltée dans le tissu cellulaire sous-cutané et dans le péritoine de Crotalus terrificus. Les caractères du parasite nous permettent de le ranger dans le genre Hastospiculum Skrj. 1923, genre que nous séparons du genre Hamatospiculum Skrj. 1916 (= Parhamatospiculum Skrjab. et Petrow 1928), en nous basant surtout sur la dimension des ailes caudales du mâle : il s'agit en effet d'une forme major de Hastospiculum onchocercum Chitwood r 933 .

\section{Brbetograptite}

Baylis (H. A.). - Filaria macrophallos Parona, and the genus Hastospiculum Skrjabin. Ann. Mag. Nat. Hist., IX, 193o, p. 672.

Baylis (H. A.) et DaubNey (R.). - Report on the parasitic nematodes in the Collection of the Zoological Survey of India. Mem. Ind. Mus. Calcutta, VII, r 922 , p. 263 .

Boulrkger (C. L.). - Report on a collection of parasites nematodes, mainly from Egypt. Part V. Filarioidea. Parasit., r928, p. 32 .

Chander (A. C.). - Some new Genera and Species of Nematode worms, Filarioidea, from Animals dying in the Calcutta Zoological Garden. Proc. U. S. Nat. Mus., LXXV, 1929 , p. r. 
CHrtwood (B. G.). - A review of the nematodes of the genus Hastospiculum, with descriptions of two new species. Proc. U. S. Nat. Mus., LXXX, r932, p. ${ }_{9}$.

Linstow (O, von). - Nematoden aus der Berliner Zoologischen Sammlung. Mitt. Zool. Mus. Berlin, I, I899, p. 3 .

Parona (C.). - Sopra alcuni Elminti di Vertebrati Birmani raccolti da Leonardo Fea. Ann. Mus. Civ. Stor. nat., Gênes, II, r889, p. 78 .

SANDGround (J. H.). - Report on the nematode parasites collected by the KelleyRoosevelts expedition to Indo-China with descriptions of several new species : Part I, Parasites of birds. Part II, Parasites of mammals. Zeitsch. f. Parasitenk., V, pp. 549-555.

Skrjabin (K. J.). - Contribution à l'étude de la faune helminthologique du Paraguay. Journ. russe de Zool., I, 1916, p. 736 .

- Hastospiculum varani, n. gen., n. sp., eine neue Filaria der Reptilien. Russ. Journ. Trop. Med., Moscou, I, 1923, p. r.

Skruabin (K. J.) et Petrow (A. M.). - The systematic position of Filaria nodulosa Rud. I819 (Parhamatospiculum n. gen.). Ann. Trop. Med., XXIII, r928, p. r6r.

- Parhamatospiculum bubicola n. sp., a new nematode from birds. Journ. Parasit., XXI, 1935, p. 9r.

Skrjabin (K. J.) et Schiкhobalova (N. P.). - Contribution au remaniement de la classification des nématodes de l'ordre des Filariata Skrjabin r9r5. Ann. Parasit., XIV, r936, p. 62.

Tubangur (M. A.). - Nematodes in the collection of the Philippine Bureau of Science. II, Filarioidea. Philipp. Journ. Sc., LV, 1934, p. Ir5.

WEHR (E. E.). - A revised classification of the nematode superfamily Filarioidea. Proc. Helm. Soc. Washington, II, r935, p. 84.

Yorke (W.) et Maplestone (P. A.). - Nematodes parasites of vertebrates. Londres, r926, pp. $395-43$ r.

Institut de parasitologie de la Faculté de médecine de Paris (Directeur : Prof. E. Brumpt). 